\title{
Chapter 14 \\ The Application of Nucleic Acid Amplification Strategies in Theranostics
}

Yanxialei Jiang

\begin{abstract}
Targeting nanoparticles equipped with diagnosis "tools" to malignant cells or tissues for optimal therapy is a popular concept of theranostics. As one of the most reliable and sensitive diagnosis "tools," nucleic acid detection is of growing practical interest with respect to molecular diagnostics of cancer and other genetic diseases. Particularly, PCR-based and other nucleic acid amplification strategies are most widely used in theranostics. This chapter aims at systematization and critical summarization of the applications of DNA- or RNA-targeted nucleic acid amplification strategies in theranostics.
\end{abstract}

\subsection{Introduction}

Cancer is the first leading cause of disease-associated death in China (Chen et al. 2016) and the second leading cause of death in the USA (Olaku, Taylor 2017) in decades. Current treatment modalities for tumors or cancers mainly focus on surgery, radiotherapy, chemotherapy, and immunotherapy, among which medicine treatment is still the most widely used therapeutic way for various types of tumors or cancers (Xiao et al. 2017). Nevertheless, typical anticancer therapies, such as radiotherapy and chemotherapy, cannot always work for the patients, even they helpful, they may not cure the patients completely (Weeks et al. 2012). Besides, radiotherapy and chemotherapy are also well known as their adverse side effects due to the treatment cannot distinguish the similarity between cancerous cells and healthy human cells (Emmenegger and Kerbel 2010; Karimi et al. 2016; Abou-Elkacem et al. 2016; Fan et al. 2016; Park et al. 2016). What's more, drug resistance, insufficient curative effect, and tumor relapse during therapeutic process are still the huge challenges when patients treated by conventional cytotoxic drugs or molecularly targeted therapeutics (Al-Lazikani et al. 2012; Yano et al. 2012), and the efficacy of typical anticancer therapies is usually limited in clinic.

\footnotetext{
Y. Jiang $(\bowtie)$

Shandong Provincial Key Laboratory of Detection Technology for Tumour Markers, College of Chemistry and Chemical Engineering, Linyi University, 276005 Linyi, People's Republic of China e-mail: jyxialei@126.com
} 
The ability to kill diseased cancerous cells while causing less harm to the normal cells is of great significance to reduce adverse side effects during therapeutic process especially in radiotherapy and chemotherapy. How to distinguish healthy cells and cancerous cells, how to achieve tumor cells specific targeting, are the vital factors in a cancer cure process. Particularly, a typical adopted strategy is to design a cancerous cells target-specific drug delivery system (DDS) that can transport an effective dosage of anticancer drugs to targeted cells, cancerous tissues or diseased area of the body (Nicolas et al. 2013; Ge and Liu 2013; Chen et al. 2014; Frandsen and Ghandehari 2012), and sustained release drug over a period of time in a controlled manner to increase survival of drugs (Bertrand and Leroux 2012).

Generally speaking, theranostics is a process combined diagnosis and therapy, popularly in cancer. Cancerous cells target-specific DDS accelerates the advancement of therapy; at the same time, more effective and reliable early tumor detection and diagnosing methods have also been put forward result from the clinical needs, which are also significant for cancer therapy in theranostics. The technology of nucleic acid amplification strategy opens up avenues for meeting this clinical diagnosis requirement in theranostics. Hybridization chain reaction (HCR), rolling-circle amplification (RCA), the strand displacement reaction (SDR), loop-mediated amplification (LAMP), and target-recycling amplification with endonuclease, exonuclease, and polymerase, and these nucleic acid amplification strategies have already become powerful tools for early diagnosis of cancer, due to their high sensitivity, excellent stability, and subtly designability, which demonstrate good prospects to meet clinical requirements, and assist DDS employed in theranostics.

\subsection{Theranostics}

“Theranostics," generally, points out emphatically the inseparability of diagnosis and therapy, the pillars of medicine (Baum and Kulkarni 2012). It means that "we know which sites require treatment and confirm that those sites have been treated." The term "theranostics" was first used by John Funkhouser at the beginning of the 1990s, while the concept of personalized medicine appeared at the same time (Choudhury and Gupta 2018). Theranostics provides a clinical transition from conventional disease trial and wrong medicine treatment to much more personalized and precision medicine time. Theranostics should have the ability to cure patients with more accurate diagnosis and more specific therapy plan.

Diagnostic and therapeutic agents can be formulated as a single theranostic platform and then can be attached to a biological ligand for cancerous cells or area targeting (Muthu et al. 2014). The use of aptamers as targeting ligands which conjugated to DDS nanoparticles as nanotheranostic system has been substantially studied (Vandghanooni et al. 2018). For example, Mosafer et al. (2017) loaded SPIONs and doxorubicin simultaneously into poly (lactic-co-glycolic acid) (PLGA) nanoparticles and then modified PLGA nanoparticles with AS1411 aptamer for tumor celltargeted theranostic purposes. It turned out that the AS1411 aptamer-conjugated 
PLGA nanoparticles enhanced its cellular uptake and cytotoxicity effect of doxorubicin in murine $\mathrm{C} 26$ cells.

Nanotechnology has been proved to be a promising platform for theranostic in recent advances. Nanoparticle-based contrast drug agents in theranostic offer improved capabilities for specific cancer targeting, high-resolution imaging, and prolonged circulation times in comparison with the custom used methods (Hahn et al. 2011). Even though theranostic has drawn world widely particular interest in cancer therapy since it shows significantly more advantages compared to typical radiotherapy and chemotherapy, its application also raises critical questions simultaneously. For example, is the optimal nanoparticles used in DDS for patients' therapy also the optimal nanoparticles used for cancer diagnostics (Dreifuss et al. 2015)?

\subsection{DDS Applied in Theranostics}

Nanotechnology has already involved in all fields of biochemical and chemical science and technology, according to its high financial investment and rapid development (Heath 2015). Simultaneously, nanotechnology breaks a new ground for drug delivery, especially for tumor-targeted drug delivery system (DDS). In 2004, National Cancer Institute (NCI) launched the NCI Alliance for Nanotechnology in Cancer to exploit the nanotechnology to fight cancer (Hull et al. 2014; Farrell et al. 2010).

Targeted DDS, as its name means, is to deliver the loaded cargos or treatment drugs to the interested site while reducing or avoiding the cargos or treatment drugs distribution to normal tissue or cells (Bae and Park 2011). For decades, several series of nanocarriers applied in DDS have been used for anticancer drugs delivery, including liposomes (Felfoul et al. 2016; Sharma and Sharma 1997; Gregoriadis and Florence 1993; Drummond et al. 2000), organic nanoparticles (Horcajada et al. 2010a, b), inorganic nanoparticles (Rotello 2008; Liong et al. 2008; Vivero-Escoto et al. 2010; Zhi et al. 2006), hybrid nanoparticles (Hrkach et al. 2012), polymeric micelles (Matsumura and Kataoka 2009; Norased et al. 2006, Bae et al. 2003), polymer-drug conjugates (Zhu et al. 2013), nanogels (Du et al. 2010; Jung-Kwon et al. 2008), as shown in Fig. 14.1.

In addition to solubilizing poorly soluble drugs and protecting drugs from cellular degradation, the drug-loaded nanocarriers in DDS could prolong the circulation time of released drugs and selectively deliver them to the targeted tumor tissue, tumor cells, or subcellular organelle (Shi et al. 2017). Besides, the released drugs from the nanocarriers can be controlled in a spatiotemporal manner, sustained release drug over a period of time, achieving their pharmacological activities only at the targeted site (Dai et al. 2012). FDA has already approved several nanotechnology-based antitumor preparations for decades, such as Doxil® (PEGylated liposomal doxorubicin (DOX)) and Abraxane ${ }^{\circledR}$ (paclitaxel (PTX) loaded albumin nanoparticles) (Hare et al. 2017).

Even though targeting nanocarriers to malignant tissues, tumor cells, or subcellular organelle to improve diagnosis and therapy is a popular concept, the literature 


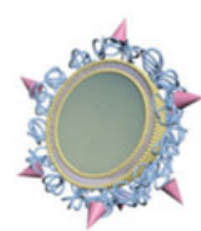

Liposome

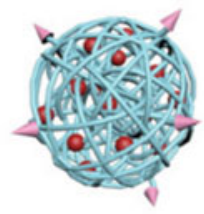

Nanoparticle

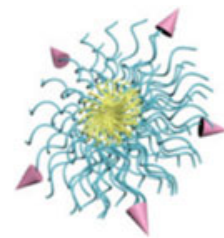

Micelle

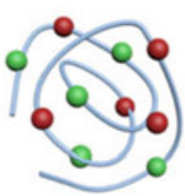

Polymer-drug
conjugate

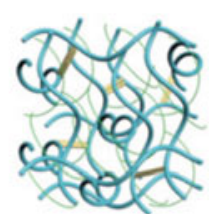

Nanogel

Fig. 14.1 Illustration of several representative nanomedicines for cancer therapy, including liposome, nanoparticle, polymer micelle, polymer-drug conjugate, and nanogel. Reprinted from Dai et al. (2017). Copyright 2017, with permission from Elsevier

survey during the past 10 years turned out that only $0.7 \%$ (median) of the administered nanocarriers is delivered to the targeted solid tumor. This has a negative effect on nanotechnology for human clinic use with respect to manufacturing, financial investment, toxicity, and imaging and therapeutic efficacy (Table 14.1) (Wilhelm et al. 2016).

\subsection{Nucleic Acid Amplification Strategies Applied in Theranostics}

For a particular disease, such as cancer, there is a growing significant interest in theranostic approaches that permit concurrent diagnosis and treatment with therapeutic development. With the increased insights into delivery kinetics and biological response, the theranostic may help open the field of medicine toward to an era of more effective and precise treatment (Peer et al. 2007; Conway et al. 2014). Unfortunately, probing and detection low-abundance cancer biomarker and transporting a high-load drug nanocarrier to specifically targeted tumor cells and, particularly, their combination still are a major challenge nowadays. Currently, theranostic approaches for probing and detection in cancer typically rely on targeting tumor-associated biomarkers with aptamer, antibodies or other ligands, which allows an accumulation of signal reporters or drug payloads in tumors and thus renders contrast imaging and selective treatment (Andersen et al. 2009). However, the lack of intelligent designs for probing and detection in diagnosis step in theranostic leads to limited imaging sensitivity and increased background interference (Bhuniya et al. 2014). The pursuit of activatable probe and detection designs represents a vital effort in cancer theranostics. 
14 The Application of Nucleic Acid Amplification ...

Table 14.1 Delivery efficiency and the number of data sets. Reprinted from Wilhelm et al. (2016), with kind permission from Springer Science + Business Media

\begin{tabular}{|c|c|c|}
\hline Category & Delivery efficiency [\%ID] ${ }^{*}$ & Number of data sets \\
\hline All data sets & 0.7 & 232 \\
\hline \multicolumn{3}{|l|}{ Year } \\
\hline 2005 & 1.4 & 8 \\
\hline 2006 & 0.7 & 8 \\
\hline 2007 & 1.0 & 24 \\
\hline 2008 & 0.3 & 8 \\
\hline 2009 & 0.9 & 11 \\
\hline 2010 & 0.8 & 14 \\
\hline 2011 & 0.7 & 27 \\
\hline 2012 & 0.7 & 14 \\
\hline 2013 & 0.5 & 35 \\
\hline 2014 & 0.8 & 38 \\
\hline 2015 & 0.5 & 45 \\
\hline \multicolumn{3}{|l|}{ Mateiral } \\
\hline Inorganic & 0.8 & 86 \\
\hline Organic & 0.6 & 137 \\
\hline \multicolumn{3}{|c|}{ Inorganic material } \\
\hline Gold & 1.0 & 45 \\
\hline Iron oxide & 0.6 & 8 \\
\hline Silica & 0.4 & 13 \\
\hline Quantum dots & 0.9 & 5 \\
\hline Other & 0.6 & 14 \\
\hline \multicolumn{3}{|c|}{ Organic material } \\
\hline Dendrimers & 1.4 & 7 \\
\hline Liposomes & 0.5 & 27 \\
\hline Polymeric & 0.6 & 62 \\
\hline Hydrogels & 0.5 & 18 \\
\hline Other & 0.9 & 23 \\
\hline \multicolumn{3}{|c|}{ Targeting strategy } \\
\hline Passive & 0.6 & 175 \\
\hline Active & 0.9 & 57 \\
\hline
\end{tabular}




\subsubsection{Theranostics Based on DNA Rolling-Circle Amplification}

Rolling-circle amplification (RCA) is an isothermal nucleic acid amplification technique, and a variety of biomarkers such as genes, microRNAs, proteins with ultra-low cellular abundance have been successfully detected using developed RCA-based probes. In recent decades, various approaches have been achieved to improve the RCA technique, and it has become an attractive detection tool for biomedical applications. In this section, I will summarize the RCA-DDS combined cancer clinical applications, making an outlook on the trends of the related research fields in the future (Wang et al. 2015a; Chen et al. 2015).

In most assays based on RCA, the quantification of the target is achieved through the RCA product quantification. The RCA products not only can turn into nanostructures by self-assembly, but also can form solubilized threads. Zhang's group synthesized MNP/DNA-SP as nanocarrier and doxorubicin embedded in MNP/DNA-SP as therapy drug to constructed a DDS (Guo et al. 2017b). By modification of different functional elements in the surface of MNP/DNA-SP, such as aptamers and disulfide linkages, the synthesized MNP/DNA-SP-DOX can be used for targeted GSH or CEM cells detection and doxorubicin delivery, resulting in the relief of cell apoptosis in target GSH or CEM cells, as shown in Fig. 14.2. For those that have not yet been screened by their aptamers specifically, functional element (e.g., FA) modified poly $\mathrm{T}$ tailed sequences were taken to MNP/DNA-SP by terminal transferase-mediated chain elongation. Different functional elements as ligands for different targets can be incorporated into MNP/DNA-SP, which can achieve versatile targeted and detection applications. The advantages of MNP/DNA-SP-DOX cargo displayed high selectivity, superior biocompatibility, and convenient drug delivery. The potent antitumor efficacy of DOX delivered by the biocompatible MNP/DNA-SP nanocarrier was also tested in a model of xenografted tumors of mice. Consequently, the result is a combination of traceable targeted detection and drug delivery that serves as a multifunctional MNP/DNA-SP, which will provide a novel theranostic platform for cancer targets detection and may also approve other clinical applications.

Considering the requirement for combination of cancer clinical diagnosis and therapy, Guo and her co-workers developed a magnetic nanoparticle (MNP)-based cellular DDS for DOX cellular load and release to kill cancerous cells (Guo et al. 2017a). As shown in Fig. 14.3, MNPs surface was modified with RNA nanoflowers (RNA NF) through biotin-avidin conjugation (MNP/RNA NF), and RNA NF was produced based on rolling-circle transcription (RCT). To realize the specific targeting cancerous cell delivery, they employed folic acid (FA) as active targeting ligands to functionalize MNP/RNA NF and constructed FA/MNP/RNA NF nanostructure. While for specific binding, folate receptors (FRs) is a wise choice for specific binding of FA, which is overexpressed on the cancer cell membrane, while low expressed in the normal cell. The anticancer drug DOX as well as the photosensitizer TMPyP4, which could generate reactive oxygen species (ROS) with $650 \mathrm{~nm}$ light, was both loaded into this FA/MNP/RNA NF nanocarrier. The result showed that 


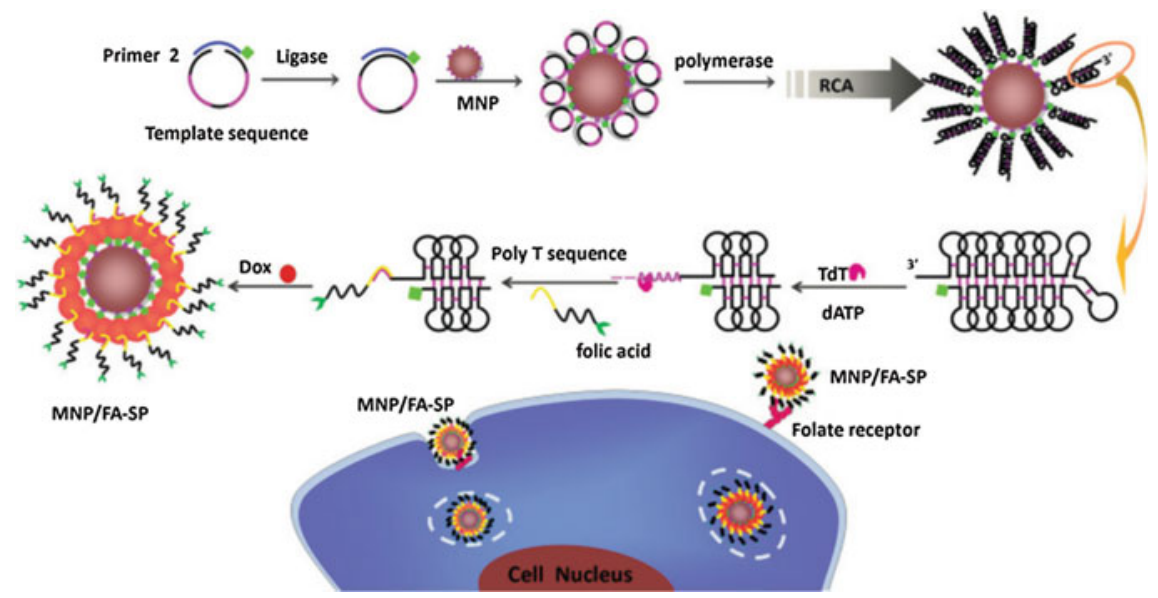

Fig. 14.2 Schematic diagram of folic acid decorated MNP/DNA-SP for cellular targeted drug delivery Reproduced from Guo et al. (2017a, b) by permission of the Royal Society of Chemistry

FA/MNP/RNA NF nanocarrier has better treatment efficacy than DOX traditional delivery way. Additionally, FA/MNP/RNA NF could also be used as a probe for HeLa cells' detection, and it can detect as low as 50 cells (Guo et al. 2017b). Thus, the nucleic acid amplification technique assisted by MNPs holds great promise for potential applications in cancer diagnostics and therapeutics.

\subsubsection{Theranostics Based on Hybridization Chain Reaction}

Hybridization chain reaction (HCR), in which stable DNA monomers assemble only upon exposure to a target DNA fragment (Dirks and Pierce 2004). Generally speaking, two stable DNA hairpins' species coexist in reaction solution and then introduce initiator strands to trigger a cascade of hybridization processes that yields nicked double helices analogous to alternating copolymers. By coupling HCR to aptamer triggers, amplification of more diverse recognition reactions can be achieved. In HCR, this functionality allows DNA to act as an amplifying transducer for biosensing applications.

Based on the feature and function of the telomerase primer and its extension products (Qian et al. 2013, 2014), Zhang and her co-workers proposed a new strategy for synthesis a novel nuclear-shell biopolymers initiated by telomere elongation and can be used for telomerase activity detection and drug delivery to cancer cells (Zhang et al. 2017). As shown in Fig. 14.4, telomerase can elongate the telomere primers, which resulting in inner chain substitution followed by the release of RCA primers and loaded drug. A core nanoball, an outer shell of RCA, biopolymers labeled with fluorescence probes constituted the nuclear-shell self-assembly body. After equipped 

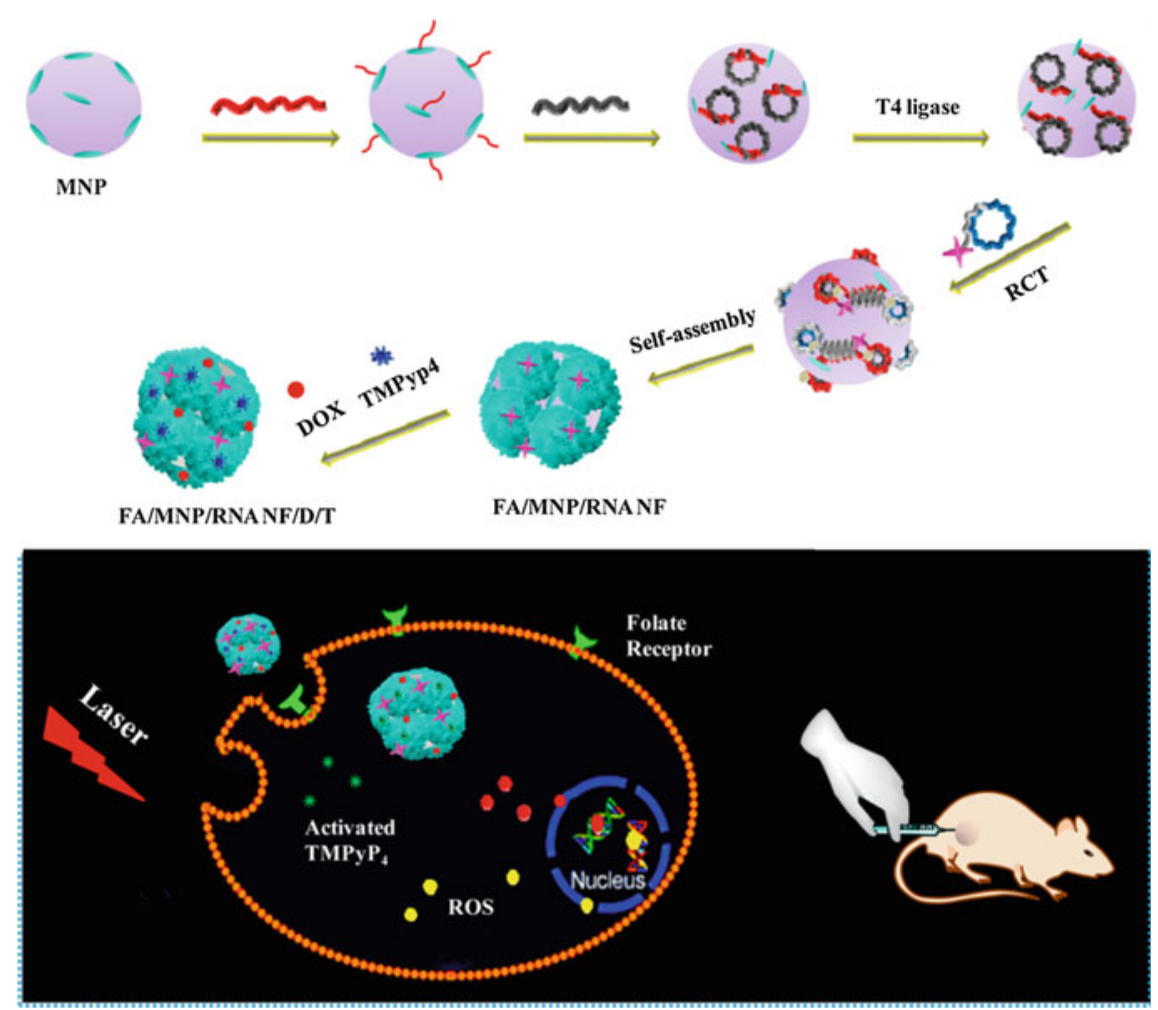

Fig. 14.3 Schematic diagram of magnetic nanoparticle-based co-delivery system Reprinted with the permission from Guo et al. (2017a, b). Copyright 2017 American Chemical Society

this platform with functionalized silica-nanoflowers (FSNFs), it was supported to detect and monitor telomerase activity and to achieve drug delivery to cancer cells. The results showed that the nuclear-shell self-assembly body, which was the product of RCA, displayed high sensitivity and activity for monitoring cellular telomerase, and efficient signal amplification effect. Consequently, as RCA product, the nuclearshell biopolymer can efficiently deliver drugs to targeted cancer cells, which reduce the undesired death of healthy cells, might prove a new way for diagnosis-treatment processes in theranostics.

By applying a structure-switching aptamer-triggered HCR on cell surface, Chu's group developed a novel activatable theranostic approach. It is the first time that achieves real-time activation and amplification for fluorescence imaging and targeting therapy use an aptamer platform (Wang et al. 2015b). The aptamer probe (AP) used in this system is designed to trigger HCR on binding to the target cell via structure switching, instead of initiating HCR in its free state, skillfully. The HCR not only amplifies fluorescence signals from a fluorescence-quenched probe (H1) for activatable tumor imaging but also accumulates high-load prodrugs from a drug-labeled 


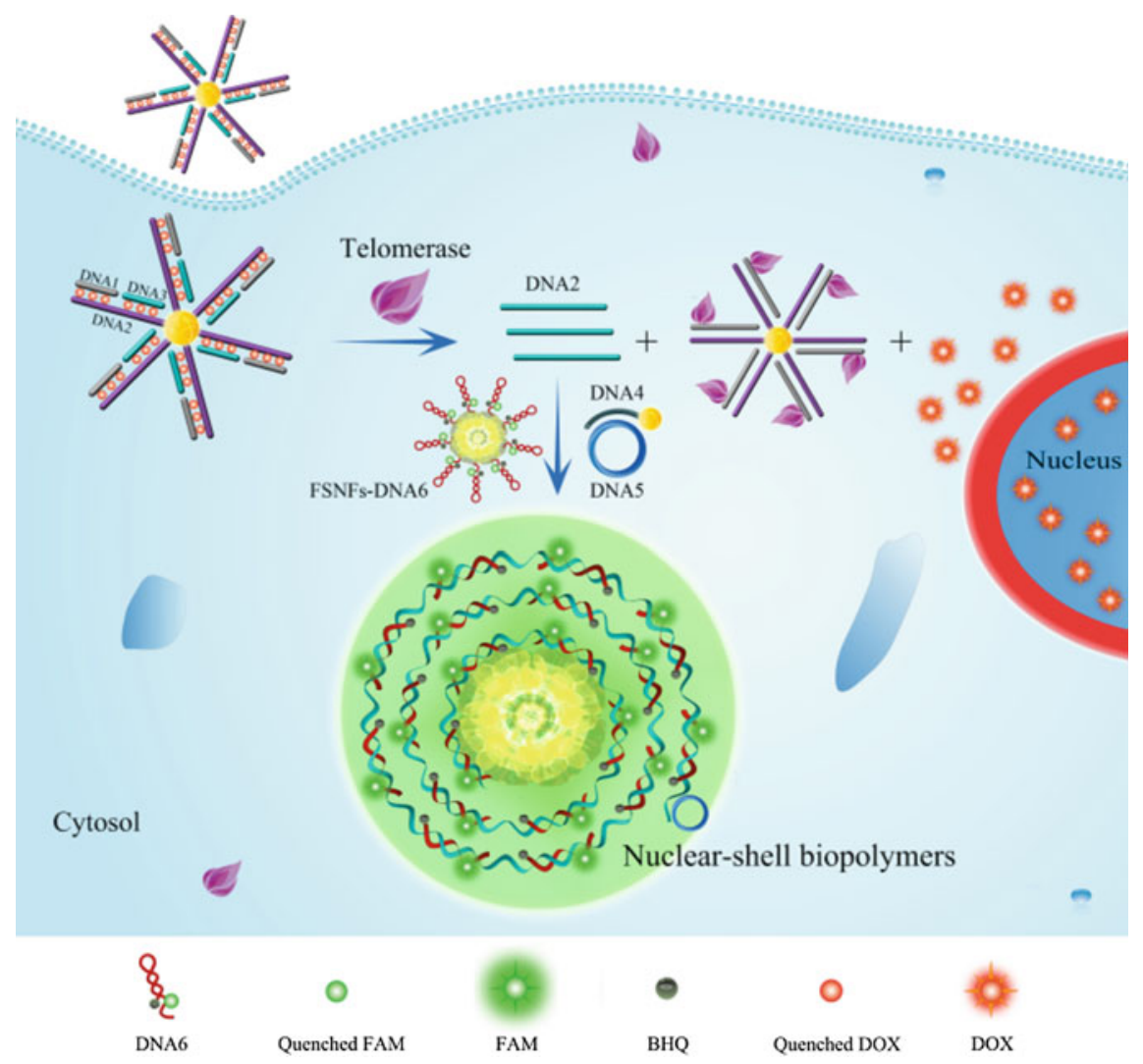

Fig. 14.4 Illustration of telomerase activity monitoring and drug delivery based on novel nuclearshell biopolymers in individual cancer cells. Reprinted with the permission from Zhang et al. (2017). Copyright 2017 American Chemical Society

probe $(\mathrm{H} 2)$ and induces its uptake and conversion into cisplatin in cells for selective tumor therapy. The in vitro result showed that this approach affords efficient signal amplification for fluorescence detection of target protein tyrosine kinase-7 (PTK7) with a detection limit of $1 \mathrm{pM}$. Live cell studies also reveal that this approach provides high-contrast fluorescence imaging and highly sensitive detection of tumor cells, while renders high-efficiency drug delivery into tumor cells via an endocytosis pathway. The results imply the potential of the HCR approach as a promising platform for early-stage disease diagnosis and precise cancer therapy (Fig. 14.5). 


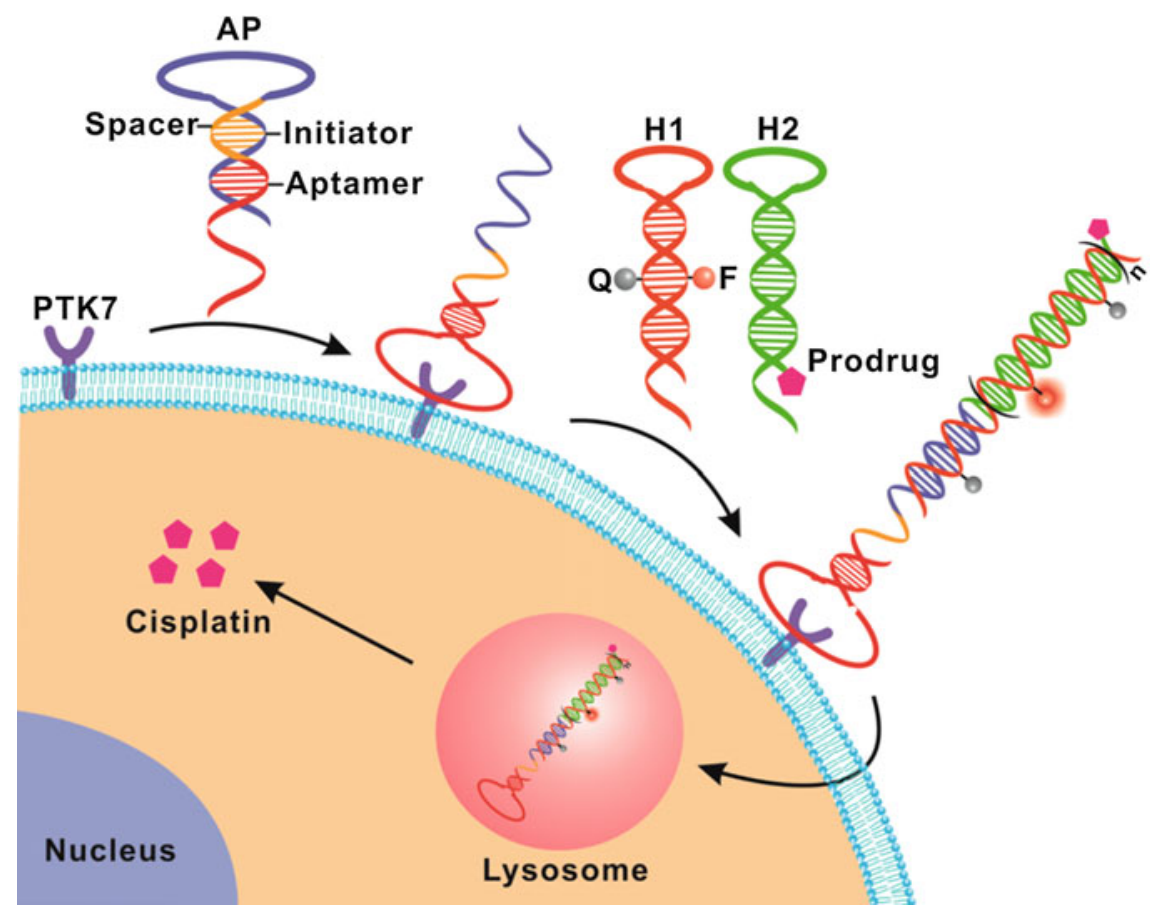

Fig. 14.5 Illustration of SATHCR for activatable theranostics. Reprinted with the permission from Wang et al. (2015a, b). Copyright 2015 American Chemical Society

\subsubsection{Theranostics Based on Aptamer-Incorporated Nucleic Acid Amplification}

Definitely, in theranostics, it is essential to monitor the expression of low-abundance biomarkers and transport a high-load drug to target cancer cells (Srinivasarao et al. 2015). Aptamers are single RNA or DNA strand with unique intramolecular conformations for selective binding to various targets. Aptamer-ligand recognition depends on the precise stacking of flat moieties, specific hydrogen bonding, and molecular shape complementarity (Bock et al. 1992; Jayasena 1999; Hermann and Patel 2000). Aptamer possesses dominant advantages over antibodies in terms of size, synthetic accessibility, and chemical modification. Therefore, aptamers are under worldwide development as a potential diagnostic or therapeutic tool in theranostics (Table 14.2) (Keefe et al. 2010).

In theranostics, the applied aptamers are functionalized with therapeutic agents or imaging probes (Wang et al. 2014; Kruspe and Hahn 2014). How to discriminate different types of cancer cells still remains challenging in the detection step due to the subtle differences in cancer cell expression of membrane receptors. Zhang's group developed a multicolor cell imaging method for distinguishing different types 
Table 14.2 Aptamers to targets of therapeutic interest. Reprinted from Keefe et al. (2010), with kind permission from Springer Science + Business Media

\begin{tabular}{|c|c|c|c|}
\hline Target (alternative name) & $K_{\mathrm{d}}(\mathrm{nM})$ & Therapeutic applications & Refs \\
\hline$\alpha$-thrombin & 25 & Prevent thrombosis & 9 \\
\hline HIV-1 reverse transcriptase & 1 & Inhibit viral replication & 134 \\
\hline HIV-1 Rev & $<1$ & Inhibit viral replication & 135 \\
\hline Fibroblast growth factor 2, basic & 0.35 & Prevent angiogenesis & 136 \\
\hline Respiratory syncytial virus & 40 & Prevent infection & 137 \\
\hline HIV-1 integrase & 10 & Inhibit viral replication & 138 \\
\hline Vascular endothelial growth factor & 0.1 & Prevent neovascularization & 139 \\
\hline Platelet-derived growth factor & 0.1 & Prevent tumor development & 83 \\
\hline Immunoglobulin $\mathrm{E}$ & 10 & Prevent allergies & 140 \\
\hline L-Selectin & 3 & Modulate inflammation & 141 \\
\hline D.Adenosine & 1100 & Unknown & 39 \\
\hline $\begin{array}{l}\text { Acetylcholine-specific } \\
\text { auto-antibodies }\end{array}$ & 60 & Treat myasthenia gravis & 142 \\
\hline Interferon- $\gamma$ & 68 & $\begin{array}{l}\text { Modulate inflammation and } \\
\text { immune response }\end{array}$ & 143 \\
\hline Keratinocyte growth factor & 0.0002 & $\begin{array}{l}\text { Treat epithelial hyperproliferative } \\
\text { disease }\end{array}$ & 144 \\
\hline Neutrophil elastase & $\mathrm{n} / \mathrm{o}$ & Modulate inflammation & 145 \\
\hline P-selectin & 0.04 & Inhibit viral adhesion & 146 \\
\hline Acetycholine receptor & 2 & Control neurotransmission & 147 \\
\hline Phospholipase $\mathrm{A}_{2}$ & 118 & Treat ARDS, septic shock & 148 \\
\hline Protein tyrosine phosphatase & 18 & $\begin{array}{l}\text { Inhibit oncogenesis, viral } \\
\text { regulation }\end{array}$ & 149 \\
\hline Activated protein $\mathrm{C}$ & 110 & Prevent thrombosis & 150 \\
\hline $\mathrm{CD} 4$ & 0.5 & Modulate immune response & 151 \\
\hline Nuclear factor $-\kappa \mathrm{B}$ & 1 & Treat chronic inflammtory disease & 152 \\
\hline $\begin{array}{l}\text { Lymphocyte function-associated } \\
\text { antigen } 1\end{array}$ & 500 & $\begin{array}{l}\text { Prevent tumor development, } \\
\text { modulate inflammation }\end{array}$ & 153 \\
\hline Cytohesin 1 & 5 & $\begin{array}{l}\text { Modulate cytoskeletal } \\
\text { reorganization }\end{array}$ & 154 \\
\hline$\alpha \vee \beta 3$ integrin & 2 & Prevent tumor development & 155,156 \\
\hline
\end{tabular}

of cancer cells with fluorophore-tagged aptamers (Fig. 14.6) (Wang et al. 2014). Aptamer sequence and aptamer-labeled dyes affect the recognition process between aptamers and cancer cells. For different cancer cell lines, even though they own the same biomarkers, when interact the fluorophore-tagged aptamers with different cancer cell lines in different degree, the result turned out that there is a distinct color to discriminate different types of cancer cells at single cell level. Based on crossreactive ability of the fluorophore-tagged aptamers, distinguish the cancer cells from 


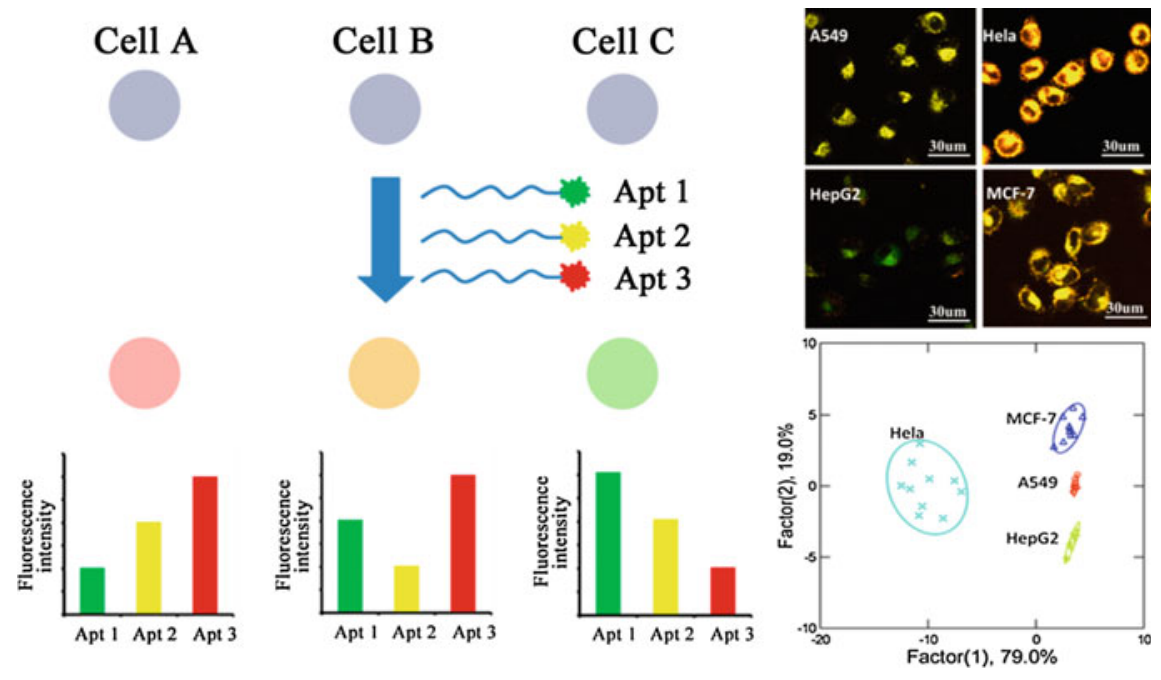

Fig. 14.6 Schematic presentation of the multicolor imaging for single cell typing with fluorophoretagged aptamers. Reprinted with the permission from Wang et al. (2014). Copyright 2014 American Chemical Society

large quantities of normal cells quickly, and identify different types of the cancer cells are achieved, simultaneously. These fluorophore-tagged aptamers have a promising application for cancer diagnostic and therapy in the future.

As one of the class of therapeutic nucleoside and nucleobase analogues drugs, 5-fluorouracil (5-FU) has been used in cancer therapy and in the treatment of various diseases for decades (Longley et al. 2003). Therefore, in DDS, nanoparticles, nanogels, and nanopolymers are mainly focus on 5-FU cellular delivery for cancer therapy (Burke et al. 2014). By applying a cell-specific cytotoxic aptamer, Sven's group found that it can be prepared in a one-step enzymatic reaction by incorporating multiple units of a nucleoside analogue directly into an aptamer that targets a cytokine receptor. In targeted cancer cells, the controlled cellular release of 5FU is initiated by intracellular nucleolytic hydrolysis of the cell-specific cytotoxic aptamer (Kruspe and Hahn 2014). Cytotoxic nucleoside and nucleobase analogues, such as 5-fluoro-2'-deoxyuridine (5-FUdR), are advantageous drugs for receptormediated active targeting as they benefit from this intracellular turnover. As showed in Fig. 14.7, 5-FUdR becomes part of the aptamer molecule itself, replacing all the uridines in the original aptamer. The controlled release of the drug inside the target cells is mediated through naturally occurred degradation by lysosomal nucleases. The hydrolysis of the aptamer would yield 5-FUdR, which could escape the lysosome by active nucleoside transporters, which normally serve as recycling gateways for lysosomal degraded nucleic acids (Fig. 14.7). 


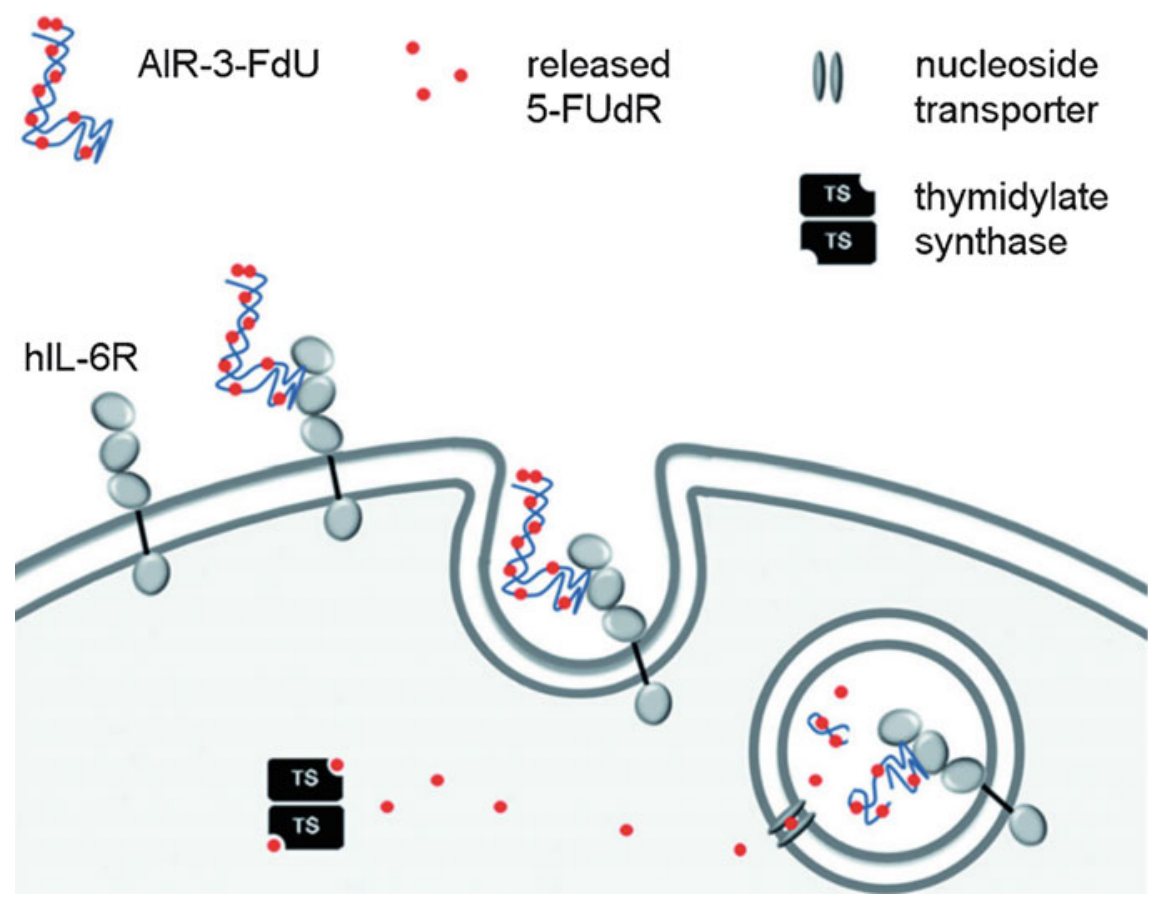

Fig. 14.7 Principle of drug delivery by AIR-3-FdU. The 5-FUdR-modified aptamer (AIR-3-FdU) binds to the human interleukin-6 receptor on the cell surface and is internalized. Reproduced from Kruspe and Hahn (2014) by permission of John Wiley\& Sons Ltd.

\subsection{Conclusion}

Theranostics is a prosperous field of cancer which combines specific targeted cancer therapy with specific cancer biomarkers-targeted diagnosis. Theranostics uses specifically diagnostic ways in the human body, to acquire diagnostic images and also to deliver a therapeutic dose of medicine to the patient. It applies nanoscience to unite diagnostic and therapeutic applications to form a single agent, allowing for diagnosis, drug delivery, and treatment response monitoring. Besides, theranostics provides a transition from conventional medicine therapy to a contemporary personalized and precision medicine approach.

In theranostics, the nucleic acid amplification strategy-based diagnostic method recognize the particular biomarkers on a tumor, amplify the signal, and allow loaded therapy drug released to specifically targets on the tumor, rather than more broadly the disease and location it presents. This contemporary form of treatment moves away from the one-medicine-fits-all and trial-and-error medicine approach, to offering the right treatment, for the right patient, at the right time, with the right dose, providing a more targeted, accurate and efficient pharmacotherapy in the form of theranostics. 
This chapter covers part of the reported applications, and many efforts have been made to develop diagnostic and theraputic assays using nucleic acid amplification combined DDS in theranostics. In the past decade, DNA- or RNA-targeted nucleic acid amplification has been well developed to help in the detection and quantification of cancer biomarkers and cells in patient-derived blood and tissue samples as well as the patient's body. While tumor is a heterogeneous tissue consisting of different types of cancer or cancer-associated cells, it may vary between different patients or even between different cancer stages in an individual patient. Therefore, currently, the discovery of new and effective diagnostic and prognostic DDS cargoes seems necessary in theranostics.

\section{References}

Abou-Elkacem L, Wilson KE, Johnson SM et al (2016) Ultrasound molecular imaging of the breast cancer neovasculature using engineered fibronectin scaffold ligands: a novel class of targeted contrast ultrasound agent. Theranostics 6:1740-1752

Al-Lazikani B, Banerji U, Workman P (2012) Combinatorial drug therapy for cancer in the postgenomic era. Nat Biotechnol 30:679-692

Andersen ES, Dong M, Nielsen MM et al (2009) Self-assembly of a nanoscale DNA box with a controllable lid. Nature 459:73-76

Bae Y, Fukushima S, Harada A et al (2003) Design of environment-sensitive supramolecular assemblies for intracellular drug delivery: polymeric micelles that are responsive to intracellular $\mathrm{pH}$ change. Angew Chem Int Ed 42:4640-4643

Bae YH, Park K (2011) Targeted drug delivery to tumors: myths, reality and possibility. J Control Release 153:198-205

Baum RP, Kulkarni HR (2012) Theranostics: from molecular imaging using Ga-68 labeled tracers and PET/CT to personalized radionuclide therapy - the bad berka experience. Theranostics 2:437-447

Bertrand N, Leroux JC (2012) The journey of a drug-carrier in the body: an anatomo-physiological perspective. J Control Release 161:152-163

Bhuniya S, Maiti S, Kim EJ et al (2014) An activatable theranostic for targeted cancer therapy and imaging. Angew Chem Int Ed 53:4469-4474

Bock LC, Griffin LC, Latham JA et al (1992) Selection of single-stranded DNA molecules that bind and inhibit human thrombin. Nature 355:564-566

Burke CW, Et Alexander, Timbie K et al (2014) Ultrasound-activated agents comprised of 5FUbearing nanoparticles bonded to microbubbles inhibit solid tumor growth and improve survival. Mol Ther 22:321-328

Chen G, Liu D, He C et al (2015) Enzymatic synthesis of periodic DNA nanoribbons for intracellular pH sensing and gene silencing. J Am Chem Soc 137:3844-3851

Chen KJ, Chaung EY, Wey SP et al (2014) Hyperthermia-mediated local drug delivery by a bubblegenerating liposomal system for tumor-specific chemotherapy. ACS Nano 8:5105-5115

Chen W, Zheng R, Baade PD et al (2016) Cancer statistics in China, 2015. CA Cancer J Clin 66:115-132

Choudhury P, Gupta M (2018) Differentiated thyroid cancer theranostics: radioiodine and beyond. Br J Radiol 91:20180136

Conway JR, Carragher NO, Timpson P (2014) Developments in preclinical cancer imaging: innovating the discovery of therapeutics. Nat Rev Cancer 14:314-328

Dai W, Jin W, Zhang J et al (2012) Spatiotemporally controlled co-delivery of anti-vasculature agent and cytotoxic drug by octreotide-modified stealth liposomes. Pharm Res 29:2902-2911 
Dai W, Wang X, Song G et al (2017) Combination antitumor therapy with targeted dual nanomedicines. Adv Drug Deliv Rev 115:23-45

Dirks RM, Pierce NA (2004) Triggered amplification by hybridization chain reaction. Proc Natl Acad Sci U S A 101:15275-15278

Dreifuss T, Betzer O, Shilo M et al (2015) A challenge for theranostics: is the optimal particle for therapy also optimal for diagnostics? Nanoscale 7:15175-15184

Drummond DC, Zignani M, Leroux JC (2000) Current status of pH-sensitive liposomes in drug delivery. Prog Lipid Res 39:409-460

Du JZ, Sun TM, Song WJ et al (2010) A tumor-acidity-activated charge-conversional nanogel as an intelligent vehicle for promoted tumoral-cell uptake and drug delivery. Angew Chem Int Ed 49:3621-3626

Emmenegger U, Kerbel RS (2010) Cancer: chemotherapy counteracted. Nature 468:637-638

Fan $\mathrm{CH}$, Cheng $\mathrm{YH}$, Ting $\mathrm{CY}$ et al (2016) Ultrasound/magnetic targeting with SPIO-DOXmicrobubble complex for image-guided drug delivery in brain tumors. Theranostics 6:1542-1556

Farrell D, Alper J, Ptak K et al (2010) Recent advances from the national cancer institute alliance for nanotechnology in cancer. ACS Nano 4:589-594

Felfoul O, Mohammadi M, Taherkhani S et al (2016) Magneto-aerotactic bacteria deliver drugcontaining nanoliposomes to tumour hypoxic regions. Nat Nanotechnol 11:941-947

Frandsen JL, Ghandehari H (2012) Recombinant protein-based polymers for advanced drug delivery. Chem Soc Rev 41:2696-2706

Ge Z, Liu S (2013) Functional block copolymer assemblies responsive to tumor and intracellular microenvironments for site-specific drug delivery and enhanced imaging performance. Chem Soc Rev 42:7289-7325

Gregoriadis G, Florence AT (1993) Liposomes in drug delivery. Drugs 45:15-28

Guo Y, Li S, Wang Y et al (2017a) Diagnosis-therapy integrative systems based on magnetic RNA nanoflowers for co-drug delivery and targeted therapy. Anal Chem 89:2267-2274

Guo Y, Wang Y, Li S et al (2017b) DNA-spheres decorated with magnetic nanocomposites based on terminal transfer reactions for versatile target detection and cellular targeted drug delivery. Chem Commun 53:4826-4829

Hahn MA, Singh AK, Sharma P et al (2011) Nanoparticles as contrast agents for in-vivo bioimaging: current status and future perspectives. Anal Bioanal Chem 399:3-27

Hare JI, Lammers T, Ashford MB et al (2017) Challenges and strategies in anti-cancer nanomedicine development: an industry perspective. Adv Drug Deliv Rev 108:25-38

Heath JR (2015) Nanotechnologies for biomedical science and translational medicine. Proc Natl Acad Sci U S A 112:14436-14443

Hermann T, Patel DJ (2000) Adaptive recognition by nucleic acid aptamers. Science 287:820-825

Horcajada P, Chalati T, Serre C et al (2010a) Porous metal-organic-framework nanoscale carriers as a potential platform for drug delivery and imaging. Nat Mater 9:172-178

Horcajada P, Serre C, Valletregí M et al (2010b) Metal-organic frameworks as efficient materials for drug delivery. Angew Chem Int Ed 45:5974-5978

Hrkach J, Von Hoff D, Mukkaram Ali M et al (2012) Preclinical development and clinical translation of a PSMA-targeted docetaxel nanoparticle with a differentiated pharmacological profile. Sci Transl Med 4:128-139

Hull LC, Farrell D, Grodzinski P (2014) Highlights of recent developments and trends in cancer nanotechnology research-view from NCI Alliance for Nanotechnology in Cancer. Biotechnol Adv 32:666-678

Jayasena SD (1999) Aptamers: an emerging class of molecules that rival antibodies in diagnostics. Clin Chem 45:1628-1650

Jun Kwon OH, Ray D, Daniel J et al (2008) The development of microgels/nanogels for drug delivery applications. Prog Polym Sci 33:448-477

Karimi M, Ghasemi A, Sahandi Zangabad P et al (2016) Smart micro/nanoparticles in stimulusresponsive drug/gene delivery systems. Chem Soc Rev 45:1457-1501

Keefe AD, Pai S, Ellington A (2010) Aptamers as therapeutics. Nat Rev Drug Discov 9:537-550 
Kruspe S, Hahn U (2014) An aptamer intrinsically comprising 5-fluoro-2'-deoxyuridine for targeted chemotherapy. Angew Chem Int Ed 53:10541-10544

Liong M, Lu J, Kovochich M et al (2008) Multifunctional inorganic nanoparticles for imaging, targeting, and drug delivery. ACS Nano 2:889-896

Longley DB, Harkin DP, Johnston PG (2003) 5-fluorouracil: mechanisms of action and clinical strategies. Nat Rev Cancer 3:330-338

Matsumura Y, Kataoka K (2009) Preclinical and clinical studies of anticancer agent-incorporating polymer micelles. Cancer Sci 100:572-579

Mosafer J, Abnous K, Tafaghodi M et al (2017) In vitro and in vivo evaluation of anti-nucleolintargeted magnetic PLGA nanoparticles loaded with doxorubicin as a theranostic agent for enhanced targeted cancer imaging and therapy. Eur J Pharm Biopharm 113:60-74

Muthu MS, Leong DT, Mei L et al (2014) Nanotheranostics—application and further development of nanomedicine strategies for advanced theranostics. Theranostics 4:660-677

Nicolas J, Mura S, Brambilla D et al (2013) Design, functionalization strategies and biomedical applications of targeted biodegradable/biocompatible polymer-based nanocarriers for drug delivery. Chem Soc Rev 42:1147-1235

Norased N, Bey E, Ren J et al (2006) Multifunctional polymeric micelles as cancer-targeted, MRIultrasensitive drug delivery systems. Nano Lett 6:2427-2430

Olaku OO, Taylor EA (2017) Cancer in the medically underserved population. Prim Care 44:87-97

Park SH, Zheng JH, Nguyen VH et al (2016) RGD peptide cell-surface display enhances the targeting and therapeutic efficacy of attenuated salmonella-mediated cancer therapy. Theranostics 6:1672-1682

Peer D, Karp JM, Hong S et al (2007) Nanocarriers as an emerging platform for cancer therapy. Nat Nanotechnol 2:751-760

Qian R, Ding L, Ju H (2013) Switchable fluorescent imaging of intracellular telomerase activity using telomerase-responsive mesoporous silica nanoparticle. J Am Chem Soc 135:13282-13285

Qian R, Ding L, Yan L et al (2014) Smart vesicle kit for in situ monitoring of intracellular telomerase activity using a telomerase-responsive probe. Anal Chem 86:8642-8648

Rotello VM (2008) Advanced drug delivery reviews theme issue: "inorganic nanoparticles in drug delivery”. Adv Drug Deliver Rev 60:1225

Sharma A, Sharma US (1997) Liposomes in drug delivery: Progress and limitations. Int J Pharmaceut 154:123-140

Shi J, Kantoff PW, Wooster R et al (2017) Cancer nanomedicine: progress, challenges and opportunities. Nat Rev Cancer 17:20-37

Srinivasarao M, Galliford CV, Low PS (2015) Principles in the design of ligand-targeted cancer therapeutics and imaging agents. Nat Rev Drug Discov 14:203-219

Vandghanooni S, Eskandani M, Barar J et al (2018) Recent advances in aptamer-armed multimodal theranostic nanosystems for imaging and targeted therapy of cancer. Eur J Pharm Sci 117:301-312

Vivero-Escoto JL, Slowing II, Trewyn BG et al (2010) Mesoporous silica nanoparticles for intracellular controlled drug delivery. Small 6:1952-1967

Wang L, Deng R, Li J (2015a) Target-fueled DNA walker for highly selective miRNA detection. Chem Sci 6:6777-6782

Wang S, Kong H, Gong X et al (2014) Multicolor imaging of cancer cells with fluorophore-tagged aptamers for single cell typing. Anal Chem 86:8261-8266

Wang YM, Wu Z, Liu SJ et al (2015b) Structure-switching aptamer triggering hybridization chain reaction on the cell surface for activatable theranostics. Anal Chem 87:6470-6474

Weeks JC, Catalano PJ, Cronin A et al (2012) Patients' expectations about effects of chemotherapy for advanced cancer. N Engl J Med 367:1616-1625

Wilhelm S, Tavares AJ, Dai Q et al (2016) Analysis of nanoparticle delivery to tumours. Nat Rev Mater 1:16014

Xiao B, Ma L, Merlin D (2017) Nanoparticle-mediated co-delivery of chemotherapeutic agent and siRNA for combination cancer therapy. Expert Opin Drug Deliv 14:65-73 
Yano S, Takeuchi S, Nakagawa T et al (2012) Ligand-triggered resistance to molecular targeted drugs in lung cancer: roles of hepatocyte growth factor and epidermal growth factor receptor ligands. Cancer Sci 103:1189-1194

Zhang Z, Jiao Y, Zhu M et al (2017) Nuclear-shell biopolymers initiated by telomere elongation for individual cancer cell imaging and drug delivery. Anal Chem 89:4320-4327

Zhi PX, Zeng QH, Gao QL et al (2006) Inorganic nanoparticles as carriers for efficient cellular delivery. Chem Eng Sci 61:1027-1040

Zhu L, Wang T, Perche F et al (2013) Enhanced anticancer activity of nanopreparation containing an MMP2-sensitive PEG-drug conjugate and cell-penetrating moiety. Proc Natl Acad Sci U S A 110:17047-17052 